\title{
Highly resilient correctors for polynomials
}

\author{
Peter Gemmell * $\quad$ Madhu Sudan ${ }^{\dagger}$
}

\begin{abstract}
We consider the problem of correcting programs that compute multivariate polynomials over large finite fields and give an efficient procedure to transform any program that computes a multivariate polynomial $f$ correctly on a $1 / 2+\delta$ fraction of its inputs $(\delta>0)$ into a randomized program that computes $f$ correctly on every input with high probability. This shows that programs computing polynomials are "resilient" to a high fraction of errors. The resilience shown in this paper is better than that of the previously known correction procedures [2, 7] and is close to the information theoretic optimum. The running time of the correction procedure is polynomial in the degree of $f$, the number of variables, and $\frac{1}{\delta}$, where calls to the incorrect program are assessed a unit cost per call. An important consequence of this result is that the $n \times n$ permanent is resilient to errors of up to $1 / 2-p(n)$ for any polynomial $p(n)$.
\end{abstract}

Keywords: Theory of computation, Program correction, Multivariate polynomials, Permanent.

\section{Introduction}

The study of self-correcting programs was independently started by [4] and [8] as a mechanism to transform programs that are usually correct into randomized programs that are always correct, with high probability. In this paper, we consider the problem of self-correcting programs that compute multivariate polynomials. Formally, the problem we consider here is the following:

\section{Problem 1}

Given:

1. A program $P$ that computes, on all but an $\epsilon$ fraction of inputs from the domain $F^{n}$, some multivariate polynomial $f$ of degree at most $d$ on its input (where $F$ is some finite field).

2. $b_{1}, b_{2}, \cdots, b_{n} \in F$

Question: What is $f\left(b_{1}, \cdots, b_{n}\right)$ ?

The main question investigated in this paper is how large $\epsilon$ can be so that the problem can still be solved efficiently (for large enough finite fields $F$ ). In the terminology of Gemmell et al. [7] this is the resilience of the class of multivariate polynomials of degree $d$. Gemmell et al. show that multivariate polynomials are resilient to an error close to $1 / 4$. The running time of their algorithm

\footnotetext{
*gemmell@vina.berkeley.edu, Department of Computer Science, 570 Evans Hall, University of California, Berkeley CA 94720. Research supported by NSF Grant CCR 88-13632.

${ }^{\dagger}$ madhu@cs.berkeley.edu, Department of Computer Science, 570 Evans Hall, University of California, Berkeley, CA 94720. Research supported by NSF PYI Grant CCR 8896202.
} 
appears sensitive to the structure of the finite field; in particular, the time bound that they prove depends on the order of certain primitive roots of unity in the field. This paper improves upon their result in two ways: our self-corrector can tolerate an error arbitrarily close to $1 / 2$ and its running time does not depend in any way upon the structure of the finite field over which the computation is performed. We do require though that the finite field be large enough i.e., its size must be $\Omega\left(\left(\frac{1}{\delta}+d\right)^{2}\right)$, where $\delta=1 / 2-\epsilon$. It may be noted that this resilience is almost optimal, in the sense that if the parameter $\epsilon$ becomes equal to half then there might be two polynomials that agree with the program at half the inputs and hence the answer is not uniquely specified.

The resilience is achieved by first reducing the problem of correcting multivariate polynomials to that of correcting univariate polynomials. This reduction is similar to the reduction of Beaver and Feigenbaum [2] in that both restrict their attention to the program's computation over a smaller subdomain, where the function can be expressed as a univariate polynomial in some input. The main improvement in our result is achieved by ensuring that on the smaller domain the program is erroneous on approximately the same fraction of the inputs as on the entire input space. Section 3 describes the details of the reduction. The second part of the correction procedure, correcting univariate polynomials when the error of the program computing the polynomial is close to half, uses an elegant technique of Berlekamp and Welch [5], which they present as part of a new mechanism for correcting Reed-Solomon codes. The main advantage of the Berlekamp-Welch mechanism is that it removes, from the running time analysis, any dependence on the structure of the finite field. We include a description of this procedure in the Appendix.

\section{Definitions}

Notation : We use $x \in_{R} \mathcal{D}$ to denote that $x$ is chosen uniformly at random from $\mathcal{D}$.

Definition 2.1 A Program $P \epsilon$-computes $f$ on the domain $\mathcal{D}$ if

$$
\operatorname{Pr}_{x \in R} \mathcal{D}[P(x) \neq f(x)]<\epsilon
$$

Definition 2.2 An $\epsilon$-self-corrector for $f$ on a domain $\mathcal{D}$ is a randomized algorithm $C$ that uses $P$ as a black box, such that if $P \epsilon$-computes $f$ on $\mathcal{D}$, then

$$
\forall x \in \mathcal{D} \quad \operatorname{Pr}\left[C^{P}(x)=f(x)\right] \geq 2 / 3
$$

(Here the probability is computed over the random coin flips of C.)

Definition 2.3 A function $f$ is $\epsilon$-resilient, over a domain $\mathcal{D}$, if there exists an $\epsilon$-self-corrector for $f$ on domain $\mathcal{D}$.

\section{Self-Correcting Multivariate Polynomials}

For $\delta>0$ and $d$ a positive integer, let $F$ be a finite field of size $\Omega\left(\left(\frac{1}{\delta}+d\right)^{2}\right)$. Let $f: F^{n} \mapsto F$ be a multivariate polynomial of degree at most $d$. We restate the problem to be solved here: 


\section{Problem 2}

Given : $P$ that $(1 / 2-\delta)$-computes $f$ and $b_{1}, b_{2}, \cdots, b_{n} \in F$.

Output : $f\left(b_{1}, b_{2}, \cdots, b_{n}\right)$.

In this section we describe a randomized reduction from Problem 2 to a problem of univariate self-correction.

We construct (by careful sampling from $F^{n}$ ) a domain $D \subset F^{n}$ parametrized by a single variable $x$ (i.e., the points in the domain $D$ are given by $\{D(x) \mid x \in F\}$ ), such that $D$ satisfies the following properties:

1. The function $f^{\prime}(x) \equiv f(D(x))$, is a polynomial of degree at most $2 d$ in $x$.

2. $\hat{b} \equiv<b_{1}, \cdots, b_{2}>\in D$; In fact we will ensure $\hat{b}=D(0)$.

3. With high probability, $P$ computes $f$ on approximately the same fraction of inputs from the domain $D$ as from the domain $F^{n}$.

The three properties listed above help us as follows: The first property ensures that we are looking at univariate polynomials over the domain $D$, while the second property makes sure that this helps us find $f\left(b_{1}, \cdots, b_{n}\right)$. The last property ensures that we do not lose too much information about $f$ during the process of the reduction.

The properties 1 and 3 are contrasting in nature. Property 1 requires the domain $D$ to be nicely structured while Property 3 is what would be expected if $D$ were a random sample of $F^{n}$. In order to get both properties we pick $D$ to be a pairwise independent sample of $F^{n}$. In particular the following sampling scheme works nicely.

Pick $\hat{\alpha}$ and $\hat{\beta}$ uniformly and randomly from $F^{n}$

$$
\begin{gathered}
D_{\hat{\alpha}, \hat{\beta}}(x) \equiv \hat{\alpha} * x^{2}+\hat{\beta} * x+\hat{b} \\
D_{\hat{\alpha}, \hat{\beta}} \equiv\left\{D_{\hat{\alpha}, \hat{\beta}}(x) \mid x \in F\right\}
\end{gathered}
$$

Each coordinate of $D_{\hat{\alpha}, \hat{\beta}}(x)$ is a polynomial of degree 2 in $x$. Hence $f^{\prime}$ is a polynomial of degree at most $2 d$ in $x$. Also $D_{\hat{\alpha}, \hat{\beta}}(0)=\hat{b}$. Thus we see that $D_{\hat{\alpha}, \hat{\beta}}$ as picked above satisfies properties 1 and $2 .^{1}$ (The following example may help clear the description above: Let $F$ be $G F(17)$ and $f\left(x_{1}, x_{2}\right)=x_{1}^{2}+x_{1} x_{2}$. Say we are interested in $f(3,4)$. Let $\hat{\alpha}=<1,2>$ and $\hat{\beta}=<3,0>$. Then $D_{\hat{\alpha}, \hat{\beta}}(x)=<x^{2}+3 x+3,2 x^{2}+4>\cdot f^{\prime}(x)=f\left(x^{2}+3 x+3,2 x^{2}+4\right)=3 x^{4}+12 x^{3}+9 x^{2}+11 x+4$ is a degree 4 polynomial in $x$. Moreover $f^{\prime}(0)=f(3,4)$. Thus $f^{\prime}, D_{\hat{\alpha}, \hat{\beta}}$ satisfy the properties 1 and 2 listed above.)

The following claim establishes that $D_{\hat{\alpha}, \hat{\beta}}$ forms a pairwise independent sample of $F^{n}$.

\footnotetext{
${ }^{1}$ The idea of substituting low-degree polynomials in a single variable for the different variables, is not a new one. In particular, this has been used by Beaver et al. [3], to reduce the number of oracles used in instance hiding schemes. The underlying property that they extract is similar. They use the fact that substitution by degree $t$-polynomials yields $t$-wise independent spaces.
} 
Claim 1 For a finite field $F$, $\hat{a_{1}}, \hat{a_{2}} \in F^{n}$, and for distinct $x_{1}, x_{2} \in F \backslash\{0\}$,

$$
\underset{\hat{\alpha}, \hat{\beta}}{\operatorname{Pr}}\left[D_{\hat{\alpha}, \hat{\beta}}\left(x_{1}\right)=\hat{a_{1}} \text { and } D_{\hat{\alpha}, \hat{\beta}}\left(x_{2}\right)=\hat{a_{2}}\right]=\frac{1}{|F|^{2 n}}
$$

Proof: For each coordinate $i \in[n]$, there exists exactly one degree 2 polynomial $p_{i}$ in $x$, such that $p_{i}(0)=b_{i}, p_{i}\left(x_{1}\right)=\left(\hat{a_{1}}\right)_{i}$ and $p_{i}\left(x_{2}\right)=\left(\hat{a_{2}}\right)_{i}$. Thus when we pick a random polynomial $p_{i}$ such that $p_{i}(0)=b_{i}$ for the $i$ th coordinate, the probability that $p_{i}\left(x_{1}\right)=\left(\hat{a_{1}}\right)_{i}$ and $p_{i}\left(x_{2}\right)=\left(\hat{a_{2}}\right)_{i}$, is $\frac{1}{|F|^{2}}$. Since the events are independent for each coordinate, we have

$$
\underset{\hat{\alpha}, \hat{\beta}}{\operatorname{Pr}}\left[D_{\hat{\alpha}, \hat{\beta}}\left(x_{1}\right)=\hat{a_{1}} \text { and } D_{\hat{\alpha}, \hat{\beta}}\left(x_{2}\right)=\hat{a_{2}}\right]=\frac{1}{|F|^{2 n}}
$$

The above claim establishes that any set $S$ of the form $S \subset\left\{D_{\hat{\alpha}, \hat{\beta}}(x) \mid x \in F \backslash\{0\}\right\}$ is a pairwise independent sample of $F^{n}$. The following lemma gives a well-known property of pairwise independent spaces. The result follows from the fact that Chebychev's inequality holds for pairwise independent random variables (cf. Luby [9]).

Lemma 2 (cf. [9]) If $S$ is a pairwise independent sample of elements from some domain $\mathcal{D}$ and $I$ maps elements of $\mathcal{D}$ to the range $\{0,1\}$. Then for positive $c$

$$
\operatorname{Pr}\left[\left|E_{x \in_{R} S}[I(x)]-E_{x \in_{R} \mathcal{D}}[I(x)]\right| \geq c / \sqrt{|S|}\right] \leq 1 / c^{2}
$$

Corollary 3 If $S \subset F^{n}$ is a pairwise independent sample of t elements from $F^{n}$, and $P(1 / 2-\delta)$ computes $f$ over the domain $F^{n}$, then the probability that $P$ computes $f$ on at least $t(1 / 2+\delta)-c \sqrt{t}$ points from $S$ is at least $1-\frac{1}{c^{2}}$.

Proof: Follows from Lemma 2 by setting $I$ as follows

$$
I(x)= \begin{cases}1 & \text { if } P(x)=f(x) \\ 0 & \text { otherwise }\end{cases}
$$

Let $k$ denote the number of points from $S$ where $P$ does not compute $f$. Then, if $t>\left(\frac{c}{\delta}+d\right)^{2}$, with probability at least $\left(1-1 / c^{2}\right)$, we have that $k<(t+d) / 2$. Thus with high probability, we can reduce the problem of self-correcting $f$ at $\hat{b}$ to the problem of finding $f^{\prime}$ (recall that $f^{\prime}(x)=f(D(x))$ ) and evaluating $f^{\prime}$ at 0 . Formally, we wish to solve the following problem:

\section{Problem 3}

Input: $t$ pairs $\left(x_{i}, y_{i}\right)$ such that for all but $k<(t+d) / 2$ values of $i, y_{i}=f^{\prime}\left(x_{i}\right)$, for some univariate polynomial $f^{\prime}$ of degree at most $2 d$.

Output: $f^{\prime}$

This problem arises in various ways in coding theory and can be solved efficiently. If the $x_{i}$ are of the form $\omega^{i}$, such that $\omega^{t}=1$, then the problem becomes one of correcting generalized BCH codes. If the $x_{i}$ 's are all the elements of $F$, then the above is the error correction problem for 
Reed-Solomon codes. In the general form as it is stated above (with no constraints on the forms of the $x_{i}$ 's), the problem can still be solved efficiently and directly due to an elegant method of Berlekamp and Welch, a description of which is included in the appendix. Combining the solution of Berlekamp and Welch with the reduction given above, we get the following theorem.

Theorem 4 For $\delta>0$, if $F$ is finite field of size $\Omega\left(\left(\frac{1}{\delta}+d\right)^{2}\right)$, and if $f$ is a multivariate polynomial in $n$ variables over $F$, then $f$ is $(1 / 2-\delta)$-resilient. The time taken by the self-corrector that achieves this resilience is polynomial in $n, \frac{1}{\delta}$ and the degree $f$.

By using Lipton's [8] observation that the permanent of an $n \times n$ matrix is a multivariate polynomial in $n^{2}$ variables of degree $n$, we get the following corollary :

Corollary 5 The $n \times n$ permanent is resilient (in time polynomial in $n$ ) to errors of $1 / 2-1 / p(n$ ) for any polynomially growing function $p(n)$.

\section{Open Problems}

We provide randomized correction procedures for multivariate polynomials which can tolerate error close to $1 / 2$. The correction works only for large finite fields. An interesting open question would be to solve this problem for much smaller finite fields (say, of constant size).

Another question of interest is the following univariate polynomial reconstruction problem. "Given a black box whose output agrees with some polynomial a constant fraction (say 10\%) of the time, is it possible to reconstruct a polynomial which agrees with the black box on $10 \%$ of its output?" Ar et al. [1] have shown a wide variety of applications to this problem. They also give a solution to this problem under the assumption that the error of the black box (on the remaining $90 \%$ of the input) is not arbitrary but in some sense structured.

A solution to the univariate reconstruction problem would also be able to improve on the following result of Feige and Lund [6]. They show that unless the polynomial heirarchy collapses, the permanent cannot be computed correctly on even an exponentially small fraction of the inputs (over large finite fields). A solution to the univariate reconstruction problem would show that unless \#P $=\mathrm{BPP}$ (a weaker assumption than that used by Feige and Lund), no program can compute the permanent correctly on $10 \%$ of the inputs (over sufficiently large finite fields).

\section{Acknowledgments}

We would like to thank Elwyn Berlekamp for his interest and the time he spent with us describing his error-correcting techniques and for his permission to include details of the technique here. We also wish to thank Manuel Blum and Umesh Vazirani for their interest which motivated most of the work here and for their time and patience in listening to our ideas. We are also thankful to Elwyn Berlekamp, Diane Hernek and Umesh Vazirani for their valuable comments on this writeup. 


\section{References}

[1] S. Ar, R. Lipton, R. Rubinfeld and M. Sudan. Reconstructing Algebraic Functions from Mixed Data. Manuscript, 1992.

[2] D. Beaver and J. Feigenbaum. Hiding Instance in Multioracle Queries. Proceedings of the 7th Symposium on Theoretical Aspects of Computer Science, Springer Verlag LNCS 415, 1990, pp. $37-48$.

[3] D. Beaver, J. Feigenbaum, J. Kilian and P. Rogaway. Security with Low Communication Overhead. Proceedings of Crypto '90, Springer Verlag LNCS 537, 1991, pp. 62-76.

[4] M. Blum, M. Luby and R. Rubinfeld. Self-Testing/Correcting with Applications to Numerical Problems. Proceedings of the 22nd ACM Symposium on Theory of Computing, 1990, pp. 73-83.

[5] E. Berlekamp and L. Welch. Error Correction of Algebraic Block Codes. US Patent Number $4,633,470$.

[6] U. Feige and C. Lund. On the Hardness of Computing the Permanent of Random Matrices. Proceedings of the 24th ACM Symposium on Theory of Computing, 1992, pp. 643-654.

[7] P. Gemmell, R. Lipton, R. Rubinfeld, M. Sudan, A. Wigderson. Self-Testing/Correcting for Polynomials and for Approximate Functions. Proceedings of the 23rd ACM Symposium on Theory of Computing, 1991, pp. 32-42.

[8] R. Lipton. New directions in testing. Distributed Computing and Cryptography, DIMACS Series in Discrete Math and Theoretical Computer Science, vol. 2, American Mathematical Society, 1991, pp. 191-202.

[9] M. Luby. A Simple Parallel Algorithm for the Maximal Independent Set Problem. SIAM Journal of Computing, vol. 15, no. 4, 1986, pp. 1036-1053. 


\section{A The Berlekamp-Welch Decoder}

This section presents the solution to the following problem first introduced by Berlekamp and Welch as part of a novel method for decoding Reed-Solomon codes.

\section{Problem 4}

Given : $m$ pairs of points $\left(x_{i}, s_{i}\right) \in F \times F$ such that there exists a polynomial $K$ of degree at most $d$ such that for all but $k$ values of $i, s_{i}=K\left(x_{i}\right)$, where $2 k+d<m$.

Question : Find $K$

Consider the following set of equations:

$$
\exists W, K \quad \operatorname{deg}(W) \leq k, \operatorname{deg}(K) \leq d, W \neq 0, \text { and } \forall i \quad W\left(x_{i}\right) * s_{i}=W\left(x_{i}\right) * K\left(x_{i}\right)
$$

Any solution $W, K$ to the above system gives a solution to Problem 4. (Notice that we can cancel $W$ from both sides of the equation to get $s_{i}=f\left(x_{i}\right)$, except when $W\left(x_{i}\right)=0$, but this can happen at most $k$ times.) Conversely, any solution $K$ to Problem 4 also gives a solution to the system of equations1. ( Let $B=\left\{x_{i} \mid s_{i} \neq f\left(x_{i}\right)\right\}$. Let $W(z)$ be the polynomial $\prod_{x \in B}(z-x)$. $W, K$ form a solution to the system 1.) Thus the problem can be reduced to the problem of finding polynomials $K$ and $W$ that satisfy (1). Now consider the following related set of constraints

$$
\exists W, N \quad \operatorname{deg}(W) \leq k, \operatorname{deg}(N) \leq k+d, W \neq 0, \text { and } \forall i \quad W\left(x_{i}\right) * s_{i}=N\left(x_{i}\right)
$$

If a solution pair $N, W$ to (2) can be found that has the additional property that $W$ divides $N$, then this would yield $K$ and $W$ that satisfy (1). Berlekamp and Welch show that all solutions to the system (2) have the same $N / W$ ratio (as rational functions) and hence if equation (2) has a solution where $W$ divides $N$, then any solution to the system (2) would yield a solution to the system (1). The following lemma establishes this invariant.

Lemma 6 Let $N, W$ and $L, U$ be two sets of solutions to (2). Then $N / W=L / U$.

Proof: For $i, 1 \leq i \leq m$, we have

$$
\begin{gathered}
L\left(x_{i}\right)=s_{i} * U\left(x_{i}\right) \quad \text { and } \quad N\left(x_{i}\right)=s_{i} * W\left(x_{i}\right) \\
\Rightarrow L\left(x_{i}\right) * W\left(x_{i}\right) * s_{i}=N\left(x_{i}\right) * U\left(x_{i}\right) * s_{i} \\
\Rightarrow L\left(x_{i}\right) * W\left(x_{i}\right)=N\left(x_{i}\right) * U\left(x_{i}\right) \quad \text { (by cancellation) }
\end{gathered}
$$

(Cancellation applies even when $s_{i}=0$ since that implies $N\left(x_{i}\right)=L\left(x_{i}\right)=0$.) But both $L * W$ and $N * U$ are polynomials of degree at most $2 k+d$ and hence if they agree on $m>2 k+d$ points they must be identical. Thus $L * W=N * U \Rightarrow L / U=N / W$

All that remains to be shown is how one obtains a pair of polynomials $W$ and $N$ that satisfy (2). To obtain this, we substitute unknowns for the coefficients of the polynomials i.e., let $W(z)=$ $\sum_{j=0}^{k} W_{j} z^{j}$ and let $N(z)=\sum_{j=0}^{k+d} N_{j} z^{j}$. To incorporate the constraint $W \neq 0$ we set $W_{k}=1$. Each constraint of the form $N\left(x_{i}\right)=s_{i} * W\left(x_{i}\right), i=1 \cdots, m$ becomes a linear constraint in the $2 k+d+1$ unknowns and a solution to this system can now be found by matrix inversion.

It may be noted that the algorithm presented here for finding $W$ and $N$ is not the most efficient known. Berlekamp and Welch [5] present an $O\left(m^{2}\right)$ algorithm for finding $N$ and $W$, but proving the correctness of the algorithm is harder. The interested reader is referred to [5] for a description of the more efficient algorithm. 


\section{B Pairwise Independent Sampling}

For a real valued random variable $Z$, let $E[Z]$ denote its expected value and let $V[Z] \equiv E\left[(Z-E[Z])^{2}\right]$ denote its variance.

Lemma 7 If $S$ is a pairwise independent sample of elements from some domain $\mathcal{D}$ and $I$ maps elements of $\mathcal{D}$ to the range $\{0,1\}$. Then for positive $c$

$$
\operatorname{Pr}\left[\left|\frac{\sum_{x \in S} I(x)}{|S|}-E[I(x)]\right| \geq c / \sqrt{|S|}\right] \leq 1 / c^{2}
$$

Proof: Since $S$ is a pairwise independent collection of elements from $\mathcal{D}$ we have

$$
E\left[\frac{\sum_{x \in S} I(x)}{|S|}\right]=E[I(x)] \text { and } V\left[\frac{\sum_{x \in S} I(x)}{|S|}\right]=\frac{V[I(x)]}{|S|}
$$

By Chebychev's inequality, we have for any random variable $Y$

$$
\operatorname{Pr}[|Y-E(Y)|>c \sqrt{V(Y)}] \leq 1 / c^{2}
$$

Applying this inequality to the variable $\frac{\sum_{x \in S} I(x)}{|S|}$, we get

$$
\operatorname{Pr}\left[\left|\frac{\sum_{x \in S} I(x)}{|S|}-E[I(x)]\right| \geq c / \sqrt{|S|}\right] \leq 1 / c^{2}
$$

